\title{
Consequences of 1BL/1RS Translocation on Agronomic and Physiological Traits in Wheat
}

\author{
S. Tahmasebi, B. Heidari* ${ }^{*}$ H. Pakniyat and A. Dadkhodaie \\ Department of Crop Production and Plant Breeding, College of Agriculture, 65186-71441, Shiraz University, \\ Shiraz, Iran
}

(Received 29 October 2014; Accepted 9 January 2015;

Communiated by A. Börner)

\begin{abstract}
The 1BL/1RS wheat-rye translocations had been used in wheat breeding programs worldwide. The objective of this study was to determine the effect of the 1BL/1RS translocation in SeriM82 /Babax recombinant population. 167 lines of this population were assayed under well-irrigated, terminal drought, heat and a combination of heat and drought stress conditions in two years. 5S rDNA and Iag95 markers were used to differentiate genotypes with or without the1BL/1RS translocation. Presence of $1 \mathrm{BL} / 1 \mathrm{RS}$ translocation reduced grain yield (YLD), grain per spike (GSP) and grain per $\mathrm{m}^{2}$ (GM2). QTLs in $1 \mathrm{BL} / 1 \mathrm{RS}$ segments indicated increased thousand-grain weight (TGW), chlorophyll content, spikelet per spike (SPLS), spike compactness (SCOM) and awn length (AWL) but reduced YLD, GSP and GM2. The 1BL/1RS carrying lines' response varied between assayed environments. Plants of drought trials were more affected by $1 \mathrm{BL} / 1 \mathrm{RS}$ compared to others. Differences in the effects of 1BL/1RS and QTLs suggest that gene expression at translocation loci is restricted to specific environmental conditions. In general, the 1BL/1RS translocation could not be a suitable source of genetic diversity for enhancing grain yield under heat and drought stresses.
\end{abstract}

Keywords: 1BL/1RS translocation, wheat, stress, QTL

\section{Introduction}

Rye (Secale cereale L.) which is an important resource of useful genes has been widely employed in wheat improvement (Jiang et al. 2010). The short arm of rye chromosome 1 (1RS) carries genes for grain yield enhancement and has been also reported as a former source of disease and insect resistance genes (McIntosh 1983; Singh et al. 1990; Rogowsky et al. 1993), stress tolerance, and wheat adaptation to environmental conditions (Rajaram et al. 1983; Schlegel and Korzun 1997). This particular 1BL/1RS chromosome translocation spontaneously arose in a wheat-rye cross progeny during the 1930s. It succeeded into different German wheat strains, until it was introgressed into foreign varieties. Famous Russian bread wheats, such as Kavkaz and Aurora were among them (Schlegel and Korzun 1997). The origin of the alien chromatin could be traced back to the

\footnotetext{
* Corresponding author; E-mail: bheidari@shirazu.ac.ir
} 
rye variety Petkus (Schlegel and Korzun 1997). This translocation carried resistance genes for pathogens responsible for disease resistance such as powdery mildew (Erysiphe graminis), stripe rust (Puccinia striiformis), leaf rust (P. triticina), and stem rust (P. graminis) (Bartos et al. 1973; Zeller 1973). Although, positive effects of 1RS/1BL on wheat biomass, spikes per square meter, grain weight, and test weight have been reported, no significant effects on grain yield were detected by other authors (Villareal et al. 1991, 1994). Previous works on SeriM82/Babax population indicated that the QTL alleles associated with 1BL/1RS translocation reduced grain yield (Mathews et al. 2008; Rattey et al. 2009; Pinto et al. 2010; McIntyre et al. 2010). Evidences show that the impact of the $1 \mathrm{BL} / 1 \mathrm{RS}$ translocation on performance is highly linked to genetic background and environmental conditions. Despite the loss of disease resistance and its negative effect on bread-making quality parameters, the $1 \mathrm{RS} / 1 \mathrm{BL}$ translocation lines continue to play a significant role in wheat breeding programs, specially, in developing countries (Dhaliwal and MacRitchie 1990; Fenn et al. 1994; Dadkhodaie et al. 2011; Peake et al. 2011; Zhao et al. 2012). For example, about $45 \%$ of commercial wheat cultivars from 17 countries from 1991 to 1995 , up to $50 \%$ of all cultivars in China (Zhou et al. 2003), 53\% of cultivars registered in Hungary (Hoffmann 2008) and 21\% of Iranian wheat cultivars (Bagherikia et al. 2014) carry the 1BL/1RS translocation.

Due to the quantitative nature of agronomic and physiological traits, concomitant use of genotyping (i.e., QTL analysis) and phenotyping of the $1 \mathrm{BL} / 1 \mathrm{RS}$ and $1 \mathrm{~B}$ lines can provide a better understanding of the effect of $1 \mathrm{BL} / 1 \mathrm{RS}$. The major objectives of this study were to (i) determine the effects of $1 \mathrm{RS} / 1 \mathrm{BL}$ translocations on agronomic, phenological and physiological traits in SeriM82 (1BL/1RS line)/Babax (1R line) population under well-irrigated, terminal drought, heat and combined heat and drought stress conditions and to (ii) compare QTL effects in $1 \mathrm{RS} / 1 \mathrm{BL}$ and $1 \mathrm{BL}$ genomic regions on wheat agronomic traits.

\section{Materials and Methods}

\section{Plant materials and field trials}

Plant materials included 167 recombinant inbred lines (RILs) developed from the cross SeriM82 $\times$ Babax that were evaluated in eight experiments in Darab Agricultural Research Station $\left(28^{\circ} 47^{\prime} \mathrm{N}, 57^{\circ} 17^{\prime} \mathrm{E}, 1110 \mathrm{~m}\right.$ altitude). This region is located at southern warm and dry area of Iran and experiences high temperatures and drought during wheat reproductive stages. SeriM82 which is a semi-dwarf spring wheat variety derived from the "Veery" cross (KVZ/BUHO//KAL/BB) while Babax released from the "Babax" cross program (BOW/NAC//VEE/3/BJY/COC) (Olivares-Villegas et al. 2007; Pinto et al. 2010). SeriM82 carries the $1 \mathrm{BL} / 1 \mathrm{RS}$ (rye) translocation, and is characterized by high yield potential. The Babax parental line is recognized as drought tolerant variety (McIntyre et al. 2010; Pinto et al. 2010).

Eight experiments were conducted in 2010-2011 and 2011-2012 growing seasons. In each experiment, RILs and parental cultivars were sown in a two-replicated $\alpha$-lattice de- 
sign. Plots were two $3.5 \mathrm{~m}$ long beds comprising three rows spaced $20 \mathrm{~cm}$. Standard crop management practices were applied, and weed and diseases were controlled in all trials. In each year, four experiments including normally irrigated condition, terminal drought stress, heat stress and combined terminal drought and heat stress trials were performed. For normally irrigated conditions, an experiment with normal sowing date (December 1) and well-irrigated conditions throughout the crop cycle was set out in each of 2010-2011 (IR11) and 2011-2012 (IR12) growing seasons. For terminal drought experiments in 2010-2011 (DR11) and 2011-2012 (DR12), the seeds were sown on December first and irrigation stopped from booting stage to end season. Two experiments with delayed sowing date on January 15 th were considered as terminal heat stressed trials in the first (HT11) and second (HT12) years. Genotypes in HT11 and HT12 experiments were normally irrigated throughout the crop cycle but faced with high end-season temperatures. In combined heat and drought stress (HD11 and HD12) trials, the seeds were sown on January 15 th and irrigation stopped from the booting stage to end season.

\section{Data collection}

The traits of interest were classified into four groups as: (1) agronomic traits [grain yield (YLD), grain number per $\mathrm{m}^{2}$ (GM2), thousand-grain weight (TGW), spikelet per spike (SPLS), grain per spike (GSP), spike length (SPL) and spike compactness (SCOM)], (2) phenological traits [day to heading (DHE) and day to maturity (DMA)], (3) physiological traits [canopy temperature at vegetative (CTv) and grain-filling (CTg) stages, chlorophyll content (SPAD)], and (4) morphological traits [plant height (PHE), leaf wax (WAX), leaf rolling (ROL) and awn length (AWL)].

DHE was calculated based on the difference of emergence date of $50 \%$ of seedlings and the date that $50 \%$ of the spikes emerged from leaf sheaths. DMA recorded at the time that $50 \%$ of the spikes lost their chlorophyll. At the grain filling stage, chlorophyll content which was expressed as SPAD unit was measured using a portable chlorophyll meter (SPAD-502 Minolta, Spectrum Technologies Inc., Plainfield, IL, USA). Canopy temperature $(\mathrm{CT})$ at the vegetative $(\mathrm{CTV})$ and grain-filling $(\mathrm{CTg})$ stages were recorded by a portable infrared thermometer (Sixth Sense LT300 IRT) (Olivares-Villegas et al. 2007). CTV was recorded at the time that the spikes were visible in $10 \%$ of the population. CTg was also measured during anthesis and late grain-filling stages (Pask et al. 2012). Glaucousness (leaf wax) was visually determined using 1 to 4 scales after anthesis (Clarke et al. 1993). ROL was evaluated via visual scores of 1-5 (O’Toole and Moya 1978). PHE, SPL, SPLS, SCOM and GSP were recorded in 10 randomly plants in each plot. Grain number was counted as per square meter. YLD was measured as $\mathrm{g} / \mathrm{m}^{2}$. TGW (g) was measured from a random sample of grains.

\section{Data analysis}

Analysis of variances of data was performed using maximum likelihood (REML) procedure in Genstat V. 15 software (Payne et al. 2012). In previous study (McIntyre et al. 
2010), two rye-specific markers (5S rDAN and Iag95) were selected to differentiate $1 \mathrm{BL} / 1 \mathrm{RS}$ carrying lines from 1B lines. For each trait, 1B chromosome classes (1BL/1RS vs. 1B) were considered as fixed factor and Best Linear Unbiased Estimates (BLUEs) were calculated from analyses within each environment. For across-environments analysis, environment and 1B chromosome classes (1BL/1RS vs. 1B) were considered as fixed factor and replicates and replicates $\times$ sub-block were considered as random. Adjusted means (BLUEs) were used to determine differences between 1BL/1RS and 1B classes using the least significant difference (LSD) test.

\section{QTL analysis}

QTL analysis was performed using molecular marker data for chromosome 1B carrying T1BL/1RS. Molecular markers consisted of 27 AFLP, 6 SSR and 10 DArT (McIntyre et al. 2010; Lopes et al. 2013). 1B-a was considered as the T1BL/1RS region (for more details see Mathews et al. 2008 and McIntyre et al. 2010). The majority of markers on 1B-a exhibited segregation distortion with approximately 75\% Babax alleles (McIntyre et al. 2010). In present study, the 1B-a linkage group was subjected to QTL analysis. QTL analysis was performed using Windows QTL Cartographer 2.5 software (Wang et al. 2007). Forward regression with five background markers, a window size of $10.0 \mathrm{cM}$ and a walk speed of $2 \mathrm{cM}$ were selected to perform composite interval mapping (CIM). Threshold values (LOD) for QTL significance were estimated via a permutation test with 1000 random re-sampling (Churchill and Doerge 1994; Doerge and Churchill 1996). Putative QTLs were defined as QTLs linked to two or more markers at LOD > 3.0 while QTLs with $2.0<\mathrm{LOD}<3.0$ were considered as suggestive. Graphical map of translocation-harboring region of 1B chromosome was generated in MapChart version 2.1 software (Voorrips 2002).

\section{Results}

\section{Variations in $1 B L / 1 R S$ and $1 B$ lines}

Combined analysis of eight environments showed that all traits except ROL were significantly affected by environmental conditions (Table S1*). Traits except SPAD and AWL were affected by the chromosome classes (1BL/1RS vs. 1B). All traits showed non-significant Env $\times 1 \mathrm{BL} / 1 \mathrm{RS}$ vs. $1 \mathrm{~B}$ interactions (Table S1).

Traits' means in all genotypes are shown in Table 1. No significant difference was observed between 1BL/1RS and 1B genotypes for CTv, CTg, DHE, DMA, PHE, WAX and ROL. Compared to 1BL/1RS lines, the 1B lines had higher YLD in irrigated (about 5\%), drought (about 10\%), heat (about 5\%), and combined stress (about 9\%) trials. Grand means of 1B lines showed higher YLD (about 8\%) than the 1BL/1RS lines over eight environments (Table 1). The 1B lines had higher GM2 in irrigated (about 6\%), drought (about 9\%), heat (about 5\%), and combined stress (about 6\%) compared to the 1BL/1RS

\footnotetext{
* Further details about the Electronic Supplementary Material (ESM) can be found at the end of the article.
} 


\begin{tabular}{|c|c|c|c|c|c|c|c|c|c|c|c|c|c|c|c|c|c|c|c|}
\hline \multirow{3}{*}{$\begin{array}{l}0 \\
0 \\
0 \\
0 \\
0 \\
0\end{array}$} & 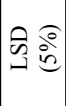 & $\begin{array}{l}n \\
\tilde{o} \\
0 \\
0\end{array}$ & ठे. & $\stackrel{7}{0}$ & $\frac{\hat{m}}{0}$ & \begin{tabular}{l}
$\infty$ \\
\multirow{n}{0}{} \\
0
\end{tabular} & nn & $\stackrel{+}{+}$ & 홍 & $\begin{array}{lll}5 & b \\
b & u \\
b & 4\end{array}$ & $\begin{array}{l}0 \\
\infty \\
\infty \\
i \\
i\end{array}$ & $\begin{array}{l}n \\
\tilde{n} \\
\tilde{o}\end{array}$ & $\mid \begin{array}{l}0 \\
0 \\
0 \\
0 \\
0\end{array}$ & 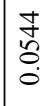 & \begin{tabular}{l} 
f \\
\multirow{0}{0}{} \\
0 \\
0
\end{tabular} & $\stackrel{n}{f}$ & $\mid \begin{array}{l}0 \\
\infty \\
0 \\
0 \\
0\end{array}$ & $\stackrel{\check{Z}}{\check{I}}$ & $\begin{array}{l}\text { के } \\
\text { के } \\
\text { ᄅun }\end{array}$ \\
\hline & $\cong$ & $\begin{array}{l}\infty \\
\infty \\
0\end{array}$ & 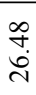 & $\begin{array}{l}\stackrel{0}{2} \\
\text { à }\end{array}$ & $\stackrel{\sim}{\stackrel{\sim}{\sim}}$ & 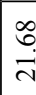 & $\begin{array}{c}\hat{\sigma} \\
\text { à }\end{array}$ & 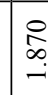 & $\frac{d}{\mathbb{Z}}$ & & ชุ & 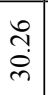 & ஃே. & \begin{tabular}{l}
0 \\
\multirow{1}{*}{}
\end{tabular} & 志 & $\begin{array}{l}\stackrel{2}{\sigma} \\
\stackrel{+}{+}\end{array}$ & م & $\begin{array}{l}8 \\
2 \\
2 \\
2\end{array}$ & 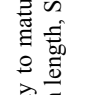 \\
\hline & $\stackrel{\cong}{\stackrel{\vartheta}{્}}$ & $\begin{array}{l}\underset{\delta}{0} \\
\stackrel{0}{-}\end{array}$ & 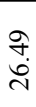 & $\begin{array}{l}\frac{\infty}{2} \\
\stackrel{a}{a}\end{array}$ & $\stackrel{m}{\oplus}$ & $\begin{array}{l}\text { ठ } \\
\text { ते }\end{array}$ & $\stackrel{?}{\dot{g}}$ & $\stackrel{n}{\infty}$ & $\frac{\infty}{\sim}$ & $\begin{array}{c}0 \\
i \\
i\end{array}$ & ל̂̊ & $\begin{array}{l}\infty \\
\dot{m} \\
\dot{m}\end{array}$ & $\begin{array}{l}\tilde{H} \\
\stackrel{2}{0} \\
0\end{array}$ & 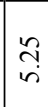 & 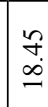 & $\begin{array}{c}\stackrel{q}{q} \\
\stackrel{\leftrightarrow}{f}\end{array}$ & $\hat{\tilde{\hat{\sigma}}}$ & 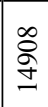 & \\
\hline \multirow{3}{*}{ 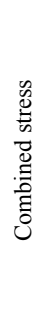 } & 雪 8 & $\stackrel{m}{0}$ & กิ & $\stackrel{m}{3}$ & 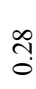 & $\stackrel{8}{\circ}$ & $\stackrel{8}{\circ}$ & $\stackrel{\infty}{\circ}$ & $\stackrel{0}{0}$ & & $\begin{array}{l}2 \\
0 \\
0 \\
0\end{array}$ & $\begin{array}{l}0 \\
n \\
0\end{array}$ & $\stackrel{0}{0}$ & $=$ & $\stackrel{0}{\stackrel{0}{0}}$ & $\underset{-}{8}$ & $\ddot{0}$ & ले & \\
\hline & $\cong$ & $\begin{array}{l}\stackrel{ }{\circ} \\
\infty \\
\infty\end{array}$ & $\hat{\grave{\rho}}$ & $\underset{\substack{\infty \\
\infty}}{\infty}$ & $\stackrel{n}{ٍ}$ & $\stackrel{f}{\tilde{n}}$ & $\underset{\infty}{\infty}$ & $\begin{array}{l}\infty \\
\infty \\
\infty \\
-\end{array}$ & $\stackrel{f}{\stackrel{f}{m}}$ & & ले & 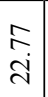 & $\stackrel{?}{q}$ & fr & $\stackrel{\Re}{\stackrel{2}{I}}$ & $\mid$\begin{tabular}{l}
0 \\
\multirow{7}{*}{} \\
$\dot{0}$ \\
n.
\end{tabular} & 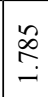 & $\begin{array}{l}\hat{\widehat{\delta}} \\
\dot{J}\end{array}$ & \\
\hline & 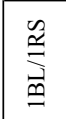 & $\begin{array}{l}\stackrel{0}{0} \\
\infty \\
\infty\end{array}$ & aे. & $\underset{\infty}{\stackrel{d}{\infty}}$ & 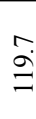 & 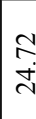 & 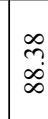 & $\underset{\lesssim}{\tilde{D}}$ & $\begin{array}{l}\infty \\
\sim \\
\sim\end{array}$ & & $\frac{\mathfrak{N}}{m}$ & $\begin{array}{l}\hat{\hat{a}} \\
\hat{\lambda}\end{array}$ & $\stackrel{\infty}{\sigma}$ & 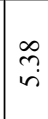 & $\begin{array}{l}\tilde{N} \\
\infty \\
\sim\end{array}$ & $\mid \begin{array}{l}\infty \\
\stackrel{+}{+} \\
\stackrel{0}{0}\end{array}$ & $\begin{array}{l}0 \\
2 \\
\infty \\
-\infty \\
-1\end{array}$ & $\begin{array}{l}\curvearrowright \\
\stackrel{0}{0}\end{array}$ & \\
\hline \multirow{3}{*}{ 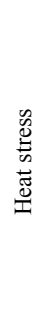 } & 目 & $=$ & तิ & กี & กิ & $\stackrel{ా}{\stackrel{0}{0}}$ & $\stackrel{\infty}{\circ}$ & : & $\stackrel{1}{\circ}$ & & $\stackrel{?}{?}$ & $\tilde{n}$ & $\stackrel{8}{0}$ & $=$ & $\stackrel{n}{0}$ & $\vec{\infty}$ & $\overline{0}$ & $\frac{n}{m}$ & \\
\hline & $\cong$ & $\begin{array}{l}\hat{b} \\
\infty \\
0\end{array}$ & $\begin{array}{l}\infty \\
\infty \\
\dot{J}\end{array}$ & 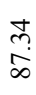 & $\overline{\check{I}}$ & $\begin{array}{l}\stackrel{+}{N} \\
\underset{\sim}{n}\end{array}$ & $\begin{array}{l}\stackrel{0}{\stackrel{8}{8}} \\
\text { a }\end{array}$ & $\stackrel{\Delta}{-}$ & ๙ิ & & $\vec{n}$ & $\begin{array}{l}\tilde{c} \\
\stackrel{\sim}{n} \\
m\end{array}$ & 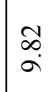 & in & 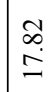 & $\mid \begin{array}{l}8 \\
\dot{j} \\
\dot{7}\end{array}$ & $\begin{array}{l}\vec{\delta} \\
\infty \\
- \\
-\end{array}$ & $\begin{array}{l}\stackrel{\partial}{\alpha} \\
\stackrel{\Delta}{=}\end{array}$ & \\
\hline & 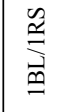 & $\begin{array}{l}\infty \\
\infty \\
\infty \\
\infty\end{array}$ & $\begin{array}{l}\stackrel{8}{+} \\
\dot{+}\end{array}$ & $\frac{\vec{\sigma}}{\dot{\infty}}$ & 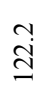 & 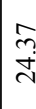 & $\stackrel{\infty}{\stackrel{\infty}{\circ}}$ & 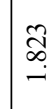 & $\frac{n}{a}$ & & I & $\begin{array}{l}\hat{0} \\
\text { in }\end{array}$ & $\overrightarrow{\widetilde{\jmath}}$ & 守 & $\begin{array}{l}n \\
n \\
\infty \\
n\end{array}$ & $\frac{?}{\stackrel{9}{\forall}}$ & 㝘 & ્ָ & \\
\hline \multirow{3}{*}{ 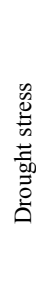 } & क्जि & oे & $\vec{ָ}$ & ?ొ? & $\stackrel{+}{?}$ & $\stackrel{7}{:}$ & $\Xi$ & oे & $\frac{n}{0}$ & & $\begin{array}{l}\infty \\
\stackrel{u}{\simeq}\end{array}$ & $\tilde{n}$ & $\frac{0}{0}$ & $\stackrel{0}{\circ}$ & $\frac{\infty}{0}$ & $\mid \begin{array}{c}0 \\
\infty \\
0\end{array}$ & $\begin{array}{l}\sigma \\
0 \\
0\end{array}$ & $\underset{\infty}{\infty}$ & \\
\hline & $\cong$ & $\begin{array}{l}8 \\
\text { in }\end{array}$ & 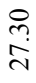 & $\stackrel{\Xi}{\Xi}$ & 导 & ㅇ. & $\stackrel{\infty}{\stackrel{\infty}{\sigma}}$ & $\hat{\overbrace{}}$ & $\bar{\lambda}$ & & $\vec{\sim}$ & $\begin{array}{l}8 \\
\check{\lambda}\end{array}$ & $\bar{m}$ & సે & 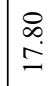 & $\mid \begin{array}{c}\infty \\
\infty \\
\dot{f} \\
\dot{f}\end{array}$ & $\underset{⿱}{\stackrel{+}{+}}$ & $\vec{n}$ & \\
\hline & $\stackrel{\mathscr{\vartheta}}{\stackrel{\vartheta}{ٍ}}$ & $\begin{array}{l}\stackrel{8}{\circ} \\
\dot{1}\end{array}$ & $\stackrel{\widetilde{n}}{\stackrel{\sim}{\sim}}$ & 三 & $\stackrel{g}{\dot{J}}$ & 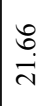 & $\stackrel{\Re}{\stackrel{2}{\sigma}}$ & $\stackrel{\circ}{\stackrel{\circ}{\rightleftharpoons}}$ & $\stackrel{f}{J}$ & & $\begin{array}{c}\infty \\
\infty \\
m\end{array}$ & $\begin{array}{l}\dot{U} \\
\dot{0} \\
\dot{ల}\end{array}$ & $\begin{array}{l}\stackrel{\hat{\imath}}{0} \\
\stackrel{0}{0}\end{array}$ & 证 & $\begin{array}{l}\stackrel{g}{+} \\
\infty \\
\stackrel{0}{\rightarrow}\end{array}$ & $\mid$\begin{tabular}{c}
0 \\
\hdashline \\
$\dot{\gamma}$
\end{tabular} & $\stackrel{2}{\stackrel{9}{-}}$ & $\underset{0}{\infty}$ & \\
\hline \multirow{3}{*}{ 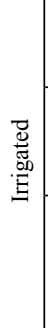 } & क्जि & oे & $\frac{ \pm}{0}$ & थै? & $\overline{3}$ & ?. & 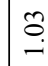 & $\stackrel{\infty}{\circ}$ & $\frac{\ddots}{0}$ & c. & $\stackrel{\sim}{\tilde{n}}$ & $\stackrel{n}{n}$ & $\stackrel{0}{0}$ & $\stackrel{\circ}{\circ}$ & $\frac{1}{0}$ & $\mid \begin{array}{l}2 \\
\infty \\
0\end{array}$ & $\begin{array}{l}0 \\
0 \\
0\end{array}$ & $\stackrel{m}{m}$ & \\
\hline & 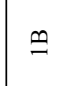 & ले & 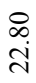 & $\vec{\Xi}$ & 홍 & $\stackrel{\text { ㅇ. }}{\circ}$ & & $\begin{array}{l}\infty \\
\stackrel{\leftrightarrow}{-}\end{array}$ & $\tilde{\Omega}$ & & ర్రి & $\begin{array}{l}\infty \\
\dot{\infty} \\
m\end{array}$ & $\begin{array}{l}\infty \\
\stackrel{1}{0} \\
\stackrel{0}{0}\end{array}$ & ले & $\stackrel{\infty}{\infty}$ & $\mid \frac{a}{\dot{n}}$ & $\underset{\mathcal{Z}}{\stackrel{2}{Z}}$ & $\begin{array}{l}0 \\
0 \\
0 \\
0\end{array}$ & \\
\hline & 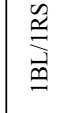 & $\begin{array}{l}\underset{+}{+} \\
\stackrel{a}{0}\end{array}$ & $\begin{array}{l}\stackrel{\imath}{i} \\
\stackrel{\Delta}{N}\end{array}$ & $\stackrel{\cong}{\Xi}$ & $\begin{array}{l}0 \\
\stackrel{8}{0} \\
\stackrel{n}{2}\end{array}$ & $\stackrel{n}{n}$ & $\stackrel{0}{\circ}$ & $\mid \begin{array}{l}: \\
\stackrel{i}{i}\end{array}$ & an & & $\overline{6}$ & $\begin{array}{l}\dot{0} \\
\text { ळे. }\end{array}$ & $\mid \begin{array}{l}n \\
\tilde{n} \\
0\end{array}$ & ì & $\begin{array}{l}\stackrel{+}{n} \\
\infty \\
\infty\end{array}$ & $\mid \begin{array}{l}\mathbb{N} \\
\dot{8} \\
i n\end{array}$ & $\stackrel{2}{\stackrel{2}{z}}$ & ڤ్ & \\
\hline & & $\vec{\theta}$ & to & $\stackrel{\underline{\underline{n}}}{\underline{\underline{n}}}$ & $\sum_{\Delta}^{\mathbb{A}}$ & is & $\stackrel{\underline{t}}{\underline{2}}$ & 㕁 & 0 & 5 & $\stackrel{\theta}{.}$ & 勇 & $\vec{\sim}$ & 学 & 足 & 芯 & $\sum_{\infty}$ & $\sum_{j}^{i}$ & \\
\hline
\end{tabular}


lines. The 1B lines also had higher GM2 than the 1BL/1RS lines in combined analysis. The 1B lines showed higher GSP in drought (about 4\%) and combined analysis (about $3.5 \%$ ), while no difference was found between two chromosome classes in other environments. The 1B lines showed higher AWL than 1BL/1RS in irrigated (about 3\%), drought (about 4\%) and combined analysis (about 4\%). No significant differences were observed between two chromosome classes in heat and combined heat and drought stress trial.

Table 2. Results of QTL analysis for wheat traits that had significant differences between 1BL/1RS and 1B lines under irrigated, heat, drought, and combined heat and drought stress trials in 2010-11 and 2011-12

\begin{tabular}{|c|c|c|c|c|c|c|c|c|}
\hline Trait & Flanking markers & $\begin{array}{l}\text { Nearer } \\
\text { marker }\end{array}$ & Allele & Position & Trial & Additive & $\mathrm{R}^{2}(\%)$ & LOD \\
\hline AWL & agc/cta-6 - agg/ctg-5 & agc/cta-6 & $1 \mathrm{~B}$ & 59.91 & DR12 & 0.1355 & 5.2 & 2.53 \\
\hline \multirow[t]{12}{*}{ SPAD } & wPt-7833 - acg/cta-2 & acg/cta-2 & 1BL/1RS & 61.51 & IR11 & 0.574 & 7.2 & 3.08 \\
\hline & aac/ctg-4 - agc/cta-2 & aac/ctg-4 & $1 \mathrm{BL} / 1 \mathrm{RS}$ & 59.21 & DR11 & 0.704 & 10.3 & 4.66 \\
\hline & agc/cta-9 - agg/ctg-3 & agc/cta-9 & 1BL/1RS & 70.41 & DR11 & 0.657 & 10.8 & 3.15 \\
\hline & wPt-5281 - aca/cac-5 & wPt-5281 & $1 \mathrm{~B}$ & 41.5 & HT11 & 0.784 & 10.3 & 2.67 \\
\hline & agg/cat-4 - agg/cat-11 & agg/cat-4 & $1 \mathrm{BL} / 1 \mathrm{RS}$ & 62.2 & HT11 & 0.626 & 6.2 & 2.70 \\
\hline & gwm273 - acc/cat-4 & acc/cat-4 & $1 \mathrm{BL} / 1 \mathrm{RS}$ & 61.6 & IR12 & 0.457 & 3 & 2.16 \\
\hline & agg/ctg-5 - acc/ctc-4 & agg/ctg-5 & $1 \mathrm{BL} / 1 \mathrm{RS}$ & 60.31 & DR12 & 0.916 & 10.7 & 4.72 \\
\hline & gwm273 - acc/cat-4 & acc/cat-4 & $1 \mathrm{BL} / 1 \mathrm{RS}$ & 61.71 & DR12 & 0.876 & 10.6 & 4.69 \\
\hline & gwm131 - agg/cat-18 & gwm131 & $1 \mathrm{BL} / 1 \mathrm{RS}$ & 64.21 & DR12 & 1.048 & 14.2 & 6.34 \\
\hline & agc/cta-9 - agg/ctg-3 & agc/cta-9 & 1BL/1RS & 70.41 & DR12 & 1.036 & 17.3 & 5.70 \\
\hline & agg/ctg-5 - acc/ctc-4 & agg/ctg-5 & 1BL/1RS & 60.3 & HT12 & 0.60 & 4.7 & 2.26 \\
\hline & agg/cac-3 - agc/cta-9 & agg/cac-3 & 1BL/1RS & 65.4 & HT12 & 0.65 & 6 & 2.90 \\
\hline \multirow[t]{13}{*}{ Yield } & aca/cac-5 - aca/caa-3 & $\mathrm{aca} / \mathrm{cac}-5$ & $1 \mathrm{~B}$ & 50.41 & IR11 & 19.51 & 9.2 & 3.84 \\
\hline & act/ctc-7 - aag/ctg-14 & act/ctc-7 & $1 \mathrm{~B}$ & 61.11 & IR11 & 21.89 & 8.2 & 4.45 \\
\hline & gwm131 - agg/cat-18 & agg/cat-18 & $1 \mathrm{~B}$ & 64.91 & IR11 & 19.08 & 7.2 & 3.45 \\
\hline & wPt-528 - aca/cac-5 & aca/cac-5 & $1 \mathrm{~B}$ & 45.51 & DR11 & 13.99 & 7.6 & 3.12 \\
\hline & gwm413 - act/ctc-5 & gwm413 & $1 \mathrm{~B}$ & 57.51 & HT11 & 13.69 & 6 & 3.36 \\
\hline & agc/cta-9 - agg/ctg-3 & agg/ctg-3 & $1 \mathrm{~B}$ & 72.40 & HT11 & 13.09 & 6.7 & 2.46 \\
\hline & gwm413 - act/ctc-5 & gwm413 & $1 \mathrm{~B}$ & 57.50 & HD11 & 9.03 & 4.6 & 2.30 \\
\hline & agc/cta-9 - agg/ctg-3 & agg/ctg-3 & $1 \mathrm{~B}$ & 72.40 & IR12 & 23.91 & 17 & 5.22 \\
\hline & agg/ctg-3 - aac/ctc-6 & aac/ctc-6 & $1 \mathrm{~B}$ & 82.61 & IR12 & 28.30 & 25.3 & 8.96 \\
\hline & aag/ctc-6 - gwm301b & gwm301b & $1 \mathrm{~B}$ & 61.8 & DR12 & 13.81 & 3.7 & 2.25 \\
\hline & agc/cag-5 - gwm131 & gwm131 & $1 \mathrm{~B}$ & 64.21 & DR12 & 15.48 & 7.7 & 3.73 \\
\hline & aca/cac-5 - aca/caa-3 & aca/cac-5 & $1 \mathrm{~B}$ & 50.41 & HT12 & 15.27 & 11.2 & 3.80 \\
\hline & agc/cag-5 - gwm131 & gwm131 & $1 \mathrm{~B}$ & 64.21 & HT12 & 17.20 & 11 & 4.96 \\
\hline \multirow[t]{8}{*}{ TGW } & act/ctc-5 - aac/ctg-4 & act/ctc-5 & $1 \mathrm{BL} / 1 \mathrm{RS}$ & 58.40 & IR11 & 0.578 & 5.2 & 2.60 \\
\hline & agg/cat-11 - aca/cac-2 & agg/cat-11 & $1 \mathrm{BL} / 1 \mathrm{RS}$ & 62.60 & IR11 & 0.592 & 5 & 2.49 \\
\hline & agg/ctg-5 - acc/ctc-4 & agg/ctg-5 & $1 \mathrm{BL} / 1 \mathrm{RS}$ & 60.31 & DR11 & 0.903 & 8.7 & 4.21 \\
\hline & agg/cat-4 - agg/cat-11 & agg/cat-4 & $1 \mathrm{BL} / 1 \mathrm{RS}$ & 62.21 & DR11 & 0.900 & 8.7 & 4.08 \\
\hline & agc/cta-9 - agg/ctg-3 & agg/ctg-3 & $1 \mathrm{BL} / 1 \mathrm{RS}$ & 76.41 & DR11 & 0.843 & 9.3 & 4.44 \\
\hline & agg/ctg-3 - aac/ctc-6 & agg/ctg-3 & 1BL/1RS & 78.60 & HD11 & 0.576 & 10.4 & 4.04 \\
\hline & act/ctc-5 - aac/ctg-4 & act $/$ ctc-5 & 1BL/1RS & 58.40 & DR12 & 0.600 & 4.4 & 2.57 \\
\hline & agg/cat-11 - aca/cac-2 & agg/cat-11 & $1 \mathrm{BL} / 1 \mathrm{RS}$ & 62.60 & DR12 & 0.627 & 3.3 & 2.11 \\
\hline
\end{tabular}


Table 2 (cont.)

\begin{tabular}{|c|c|c|c|c|c|c|c|c|}
\hline Trait & Flanking markers & $\begin{array}{c}\text { Marker with } \\
\text { max effect }\end{array}$ & Allele & Position & Trial & Additive & $\mathrm{R}^{2}(\%)$ & LOD \\
\hline \multirow[t]{14}{*}{ SPLS } & $\mathrm{aca} / \mathrm{cac}-5-\mathrm{aca} / \mathrm{caa}-3$ & aca/cac-5 & $1 \mathrm{BL} / 1 \mathrm{RS}$ & 52.40 & IR11 & 0.198 & 6.5 & 2.54 \\
\hline & agg/cat-4 - agg/cat-11 & agg/cat-4 & $1 \mathrm{BL} / 1 \mathrm{RS}$ & 62.20 & IR11 & 0.206 & 5.4 & 2.63 \\
\hline & aca/cac-5 - aca/caa-3 & aca/cac-5 & 1BL/1RS & 50.41 & HT11 & 0.209 & 8.3 & 3.26 \\
\hline & aac/cta-5 - wPt-1781 & aac/cta-5 & 1BL/1RS & 61.01 & HT11 & 0.231 & 8.2 & 4.16 \\
\hline & agc/cta-9 - agg/ctg-3 & agg/ctg-3 & $1 \mathrm{BL} / 1 \mathrm{RS}$ & 72.40 & HT11 & 0.211 & 8.1 & 2.73 \\
\hline & agc/cta-2 - aca/cta-9 & agc/cta-2 & $1 \mathrm{BL} / 1 \mathrm{RS}$ & 59.51 & HD11 & 0.217 & 7.2 & 4.01 \\
\hline & agc/cta-9 - agg/ctg-3 & agc/cta-9 & $1 \mathrm{BL} / 1 \mathrm{RS}$ & 70.40 & HD11 & 0.214 & 8.3 & 3.07 \\
\hline & aca/cac-5 - aca/caa-3 & aca/caa-3 & $1 \mathrm{BL} / 1 \mathrm{RS}$ & 54.40 & HT12 & 0.260 & 8.2 & 3.05 \\
\hline & wPt-2052 - wPt-0170 & wPt-0170 & $1 \mathrm{BL} / 1 \mathrm{RS}$ & 61.21 & HT12 & 0.297 & 7.7 & 3.76 \\
\hline & agg/cac-3 - agc/cta-9 & agg/cac-3 & $1 \mathrm{BL} / 1 \mathrm{RS}$ & 65.41 & HT12 & 0.294 & 8.7 & 3.83 \\
\hline & $\mathrm{aca} / \mathrm{cac}-5-\mathrm{aca} / \mathrm{caa}-3$ & aca/caa-3 & 1BL/1RS & 54.40 & HD12 & 0.238 & 6.3 & 2.32 \\
\hline & aca/cta-9 - agc/cta-6 & aca/cta-9 & 1BL/1RS & 59.70 & HD12 & 0.244 & 5.8 & 2.69 \\
\hline & agg/cac-3 - agc/cta-9 & agg/cac-3 & $1 \mathrm{BL} / 1 \mathrm{RS}$ & 65.41 & HD12 & 0.288 & 7.7 & 3.64 \\
\hline & agc/cta-9 - agg/ctg-3 & agg/ctg-3 & $1 \mathrm{BL} / 1 \mathrm{RS}$ & 72.40 & HD12 & 0.258 & 7.6 & 2.69 \\
\hline \multirow[t]{7}{*}{ SCOM } & aac/caa-4 - wPt-1403 & wPt-1403 & $1 \mathrm{BL} / 1 \mathrm{RS}$ & 24.60 & HT11 & 0.015 & 3.7 & 2.25 \\
\hline & agg/ctg-3 - aac/ctc-6 & aac/ctc-6 & $1 \mathrm{BL} / 1 \mathrm{RS}$ & 80.60 & HT11 & 0.016 & 4.6 & 2.13 \\
\hline & act/ctc-7 - aag/ctg-14 & act/ctc-7 & $1 \mathrm{BL} / 1 \mathrm{RS}$ & 61.11 & IR12 & 0.032 & 10 & 4.70 \\
\hline & agg/cat-11 - aca/cac-2 & agg/cat-11 & $1 \mathrm{BL} / 1 \mathrm{RS}$ & 62.61 & IR12 & 0.029 & 9.2 & 3.84 \\
\hline & agg/ctg-3 - aac/ctc-6 & agg/ctg-3 & $1 \mathrm{BL} / 1 \mathrm{RS}$ & 78.60 & DR11 & 0.022 & 6.3 & 2.14 \\
\hline & aca/cac-5 - aca/caa-3 & aca/caa-3 & 1BL/1RS & 54.40 & HT12 & 0.021 & 6.4 & 2.62 \\
\hline & gwm301b - agg/cat-4 & gwm301b & $1 \mathrm{BL} / 1 \mathrm{RS}$ & 61.90 & HT12 & 0.022 & 57 & 3.01 \\
\hline \multirow[t]{4}{*}{ GSP } & act/ctc-5 - aac/ctg-4 & act/ctc-5 & $1 \mathrm{~B}$ & 58.40 & DR11 & 1.00 & 5.3 & 2.45 \\
\hline & aca/cac-5 - aca/caa-3 & aca/cac-5 & $1 \mathrm{~B}$ & 50.40 & DR12 & 1.20 & 5.8 & 2.13 \\
\hline & agc/cta-6 - agg/ctg-5 & agc/cta-6 & $1 \mathrm{~B}$ & 59.91 & DR12 & 1.64 & 8.2 & 3.73 \\
\hline & agg/cat-11 - aca/cac-2 & agg/cat-11 & $1 \mathrm{~B}$ & 62.61 & DR12 & 1.56 & 7.8 & 3.52 \\
\hline \multirow[t]{12}{*}{ GM2 } & aca/cac-5 - aca/caa-3 & aca/cac-5 & $1 \mathrm{~B}$ & 50.41 & IR11 & 585.87 & 12 & 5.02 \\
\hline & aca/cta-9 - agc/cta-6 & aca/cta-9 & $1 \mathrm{~B}$ & 59.71 & IR11 & 621.31 & 12 & 5.68 \\
\hline & agc/cta-9 - agg/ctg-3 & agc/cta-9 & $1 \mathrm{~B}$ & 68.41 & IR11 & 553.29 & 10 & 3.70 \\
\hline & aac/ctg-4 - agc/cta-2 & aac/ctg-4 & $1 \mathrm{~B}$ & 59.21 & HT11 & 490.47 & 9 & 3.76 \\
\hline & agc/cta-9 - agg/ctg-3 & agg/ctg-3 & $1 \mathrm{~B}$ & 76.40 & HT11 & 369.14 & 6.4 & 2.75 \\
\hline & aac/ctg-4 - agc/cta-2 & aac/ctg-4 & $1 \mathrm{~B}$ & 59.21 & HD11 & 493.30 & 9 & 4.42 \\
\hline & agc/cag-5 - gwm131 & gwm131 & $1 \mathrm{~B}$ & 64.20 & HD11 & 414.70 & 6.4 & 2.90 \\
\hline & agc/cta-9 - agg/ctg-3 & agg/ctg-3 & $1 \mathrm{~B}$ & 72.40 & IR12 & 510.23 & 8.1 & 2.79 \\
\hline & agg/ctg-3 - aac/ctc-6 & aac/ctc-6 & $1 \mathrm{~B}$ & 82.61 & IR12 & 627.39 & 13 & 4.89 \\
\hline & aca/cac-5 - aca/caa-3 & aca/cac-5 & $1 \mathrm{~B}$ & 52.41 & DR12 & 618.60 & 13 & 4.40 \\
\hline & barc065 - wPt-7833 & wPt-7833 & $1 \mathrm{~B}$ & 61.41 & HT12 & 538.40 & 7 & 3.76 \\
\hline & agc/cta-9 - agg/ctg-3 & agc/cta-9 & $1 \mathrm{~B}$ & 70.40 & HT12 & 445.62 & 6.9 & 2.29 \\
\hline
\end{tabular}

AWL: awn length, SPAD: chlorophyll content unit, YLD: grain yield, TGW: thousand-grain weight, SPLS: spikelet per spike, GSP: grain per spike, SCOM: spike compactness and GM2: grain per square meter. IR11: normally irrigated trial in 2010-2011, IR12: normally irrigated trial in 2011-2012, DR11: drought stress trial in 2010-2011, DR12: drought stress trial in 2011-2012, HT11: heat stress trial in 2010-2011, HT12: heat stress trial in 2011-2012, HD11: combined stress trial in 2010-2011, HD12: combined stress trial in 2011-2012. 
The 1BL/1RS lines showed higher SPAD, SPLS and SCOM than the 1B lines in all environments. Compared to $1 \mathrm{~B}$ lines, SPAD was approximately $8 \%$ higher in $1 \mathrm{BL} / 1 \mathrm{RS}$ lines in both irrigated and drought, and about $5 \%$ in both heat and combined trials. The 1BL/1RS lines also had higher grand mean for SPAD. The 1BL/1RS lines had higher SPLS than 1B lines in irrigated, drought, heat and combined heat and drought stress trials (Table 1). The SPLS of the 1BL/1RS lines was significantly higher than 1B lines. The 1BL/1RS lines had a higher SCOM than the 1BL/1RS lines in all environments (Table 1). The $1 \mathrm{BL} / 1 \mathrm{RS}$ lines showed higher SPL in heat $(4 \%)$, combined stress $(6 \%)$ and combined analysis $(4 \%)$, while no difference was found between two chromosome classes in other environments (Table 1).

\section{$1 B L / 1 R S Q T L S$}

Total 1BL/1RS region length was about $64 \mathrm{cM}$ with $1.5 \mathrm{cM}$ average marker distance. QTLs for the traits that were significant between 1BL/1RS and 1B chromosome classes are shown in Table 2. All significant QTL alleles of 1BL/1RS except a QTL in the wPt5281 - aca/cac-5 interval in HT11 increased SPAD. Most SPAD QTLs in studied environments were detected between $59-71 \mathrm{cM}$ interval of LG 1B. A major and environmentally stable QTL (LOD = 3.15 in 2011 and 5.7 in 2012) was detected at agc/cta-9 - agg/ ctg-3 interval with increasing allele derived from SeriM82 (1BL/1RS region).

YLD increasing QTL alleles were derived from Babax (1B) and explained 3.7 to $25.3 \%$ of the YLD variation. A putative QTL, linked to aca/cac-5, explained about 9.2, 7.6 and $11.2 \%$ of YLD variation in IR11, DR11 and HT11, respectively. Another putative QTL from Babax explained 7.7 and 11\% of YLD variation in DR12 and HT12, respectively. Two QTLs explaining 17 and $25.3 \%$ of variation were detected in IR12 environment. QTL alleles from Babax parent (1B) increased TGW. Three putative QTLs that were identified in DR11 explained 8.7, 8.7 and 9.3\% of TGW variation, respectively. The estimated position of co-located QTLs for traits on translocation-harboring region is presented in Fig. S1. The QTL positioned at $76.41 \mathrm{cM}$ distance was co-located with a strong QTL for increased YLD from Babax in IR12. The putative QTL near agg/ctg-3 explained 9.3 and $10.4 \%$ of TGW variation in HT11 and HD11, respectively. A putative QTL positioned at $62.2 \mathrm{cM}$ distance had increasing effect on TGW in DR11 and it was co-located with a suggestive increasing SPAD QTL (Fig. S1).

Alleles from SeriM82 (1BL/1RS) increased SPLS. A putative QTL linked to agg/cac-3 explained 8.7 and $7.7 \%$ of SPLS variation in HT12 and DH12, respectively. This QTL was co-located with an increasing SPAD QTL in HT12. Another SPLS QTL (at $70.4 \mathrm{cM}$ distance) detected in HD11 was co-located with a putative increasing SPAD QTL in DR11 and DR12 (Fig. S1). The 1BL/1RS translocation increased SCOM in all trials. QTLs linked to act/ctc-7 and agg/cat-11 explained about 10 and 9.2\% of SCOM variation in IR12, respectively. Another putative QTL from SeriM82 detected at $54.4 \mathrm{cM}$ distance was co-located with an increasing SPLS QTL from SeriM82 in HT12 (Fig. S1). The QTL positioned at $62.6 \mathrm{cM}$ distance increased SCOM in IR12 and co-located with two TGW QTLs in IR11 and DR12 trials (Fig. S1). 
The QTL alleles from 1BL/1RS increased GSP. Two putative QTLs positioned at 59.9 and $62.6 \mathrm{cM}$ distances were identified in DR12. These two QTLs explained 8.2 and $7.8 \%$ of GSP variation, respectively. The QTL positioned at $62.6 \mathrm{cM}$ distance was co-located with a putative QTL for decreased SCOM in IR12 and a suggestive decreasing TGW QTL in IR11 and DR12. The act/ctc-5 marker at $58.41 \mathrm{cM}$ distance linked to a suggestive GSP QTL in DR11 was also linked to a suggestive decreasing TGW QTL from Babax in IR11 and DR12 (Fig. S1). Alleles from Babax (1B) increased GM2 and explained 6.4 to $13 \%$ of phenotypic variation (Table 2 ). The aac/ctg-4 marker at $59.16 \mathrm{cM}$ distance linked to a putative QTL that explained about 9\% of GM2 variation in HT11 and HD11. This QTL was co-located with a strong QTL derived from SeriM82 (1BL/1RS allele) for increasing SPAD in DR11 (Fig. S1). Another putative GM2 QTL (LOD=5.3) that was found in IR11 was co-located with a Babax QTL for increasing YLD in IR11 and HT12 and with another Babax QTL for increased GSP in DR12 (Fig. S1). The aac/ctc-6 marker at $84.98 \mathrm{cM}$ distance was co-segregated with a QTL for GM2. This putative QTL was co-located with a strong QTL for increasing YLD $(\mathrm{LOD}=8.96)$ in IR12. A robust QTL at $59.7 \mathrm{cM}$ distance for increased GM2 in IR11 was co-located with a putative QTL for increased GSP in DR12 (Fig. S1).

The only suggestive QTL for AWL was identified at $59.91 \mathrm{cM}$ distance in DR12. The effect of this QTL was attributed to $1 B L / 1 R S$ and higher AWL. The AWL QTL derived from Babax was co-located with a suggestive GSP QTL in DR12 (Table 2).

\section{Discussion}

The effect of 1BL/1RS on wheat agronomic traits has been reported (Rajaram et al. 1983; Villareal et al. 1991, 1994, 1995; Moreno-Sevilla et al. 1995; Monneveux et al. 2003; Peake 2003; Peake et al. 2011). Our result showed the significant effect of chromosome classes (1BL/1RS vs.1B) on most of the agronomic traits but some of traits were not significantly affected by $1 \mathrm{BL} / 1 \mathrm{RS}$. The $1 \mathrm{BL} / 1 \mathrm{RS}$ translocation decreased YLD in all environments which is in agreement with other reports (Mathews et al. 2008; Rattey et al. 2009; McIntyre et al. 2010; Pinto et al. 2010). In some studies, 1BL/1RS translocation increased YLD (Carver and Rayburn 1994; Villareal et al. 1994, 1995; Moreno-Sevilla et al. 1995). However, our results showed considerable negative effect of 1BL/1RS translocation on YLD in drought and combined stress trials. This shows that the effect of the $1 \mathrm{BL} / 1 \mathrm{RS}$ translocation varies upon environment. Our results were similar to those obtained by Monneveux et al. (2003) and Peake et al. (2011). Ehdaie et al. (2003) showed that the 1BL/1RS translocation caused non-significant decreases in grain yield and grain number. Present study showed that the negative effect of the 1BL/1RS translocation on YLD was associated with decreased GSP and GM2.

The 1BL/1RS lines had higher TGW than the 1B lines in irrigated, drought and combined analysis. This is in agreement with other studies (Rattey et al. 2009; McIntyre et al. 2010; Peake et al. 2011). In addition, increased TGW in 1BL/1RS lines was accompanied by increased SPAD and AWL. However, in wheat where grain-filling is mostly sink-limited (Borrás et al. 2004), reductions in grain number cannot be compensated by grain 
weight (Calderini et al. 2001; Ugarte et al. 2007). Despite increased TGW in present study, 1BL/1RS class had lower grain yield due to decreased grain number. Similarly, Peake et al. (2011) by performing a cross between SeriM82 and Hartog suggested that increased grain weight of the $1 \mathrm{BL} / 1 \mathrm{RS}$ class may be a compensatory source/sink response to decreased grain number.

QTLs on 1BL/1RS increased SPAD, TGW, SPLS, SCOM and AWL and reduced GSP, GM2 and YLD. McIntyre et al. (2010) detected a number of QTLs in 1BL/1RS region from the SeriM82 (rye) allele which increased grain weight but decreased grain number, and grain yield in low yielding environments. Pinto et al. (2010) also found Seri M82derived QTLs on translocation region that decreased grain per $\mathrm{m}^{2}$ and grain yield. Colocation of QTLs of some of traits conveys the pleiotropic or linkage effects. For instance, YLD QTLs were co-located with QTLs for GM2, SPLS, SPAD and SCOM.

In Australia, the use of wheat cultivars carrying 1RS/1BL translocation has been limited to feed and biscuit because of low bread-making qualities (i.e. sticky dough) (McIntosh et al. 2001). In contrast, wheat genotypes with this translocation have been used widely in developing countries, and are also present in soft red winter wheat in the US and Europe (Dadkhodaie et al. 2011). The frequency of 1BL/1RS translocation reached approximately to $70 \%$ at one stage in CIMMYT's spring wheat germplasm but has declined to about $30 \%$ in more recent advanced lines. The wheat cultivars Veery, Kauz and Super Kauz which carry 1BL/1RS translocation (Rabinovich 1998), are present in the pedigree of many CIMMYT lines (Dadkhodaie et al. 2011). Furthermore, the presence of the rust resistance genes $\operatorname{Lr} 26, \mathrm{Yr} 9$ and $S r 31$ also indicated that almost $60 \%$ of CIMMYT-derived germplasm carry this translocation (Dadkhodaie et al. 2011). Based on pedigree information, many of Iranian cultivars released in recent years have originated form CIMMYT or local by CIMMYT crosses. Because of negative effect of 1BL/1RS translocation on grain yield and also bread-making quality and loss of disease resistance (Dhaliwal and MacRitchie 1990; Fenn et al. 1994; Dadkhodaie et al. 2011; Peake et al. 2011; Zhao et al. 2012), in the case of using 1BL/1RS carrying lines in producing populations, selection should be made for progenies lacking this translocation.

Results showed that the $1 \mathrm{BL} / 1 \mathrm{RS}$ translocation affected grain yield and its components, AWL and SPAD. 1BL/1RS translocation reduced grain yield, grain number $/ \mathrm{m}^{2}$ and grain per spike. This negative effect on grain yield was found in heat and drought stress trial. The impact of 1BL/1RS translocation on traits varied upon the environment. Traits in drought stress trials were more affected by $1 \mathrm{BL} / 1 \mathrm{RS}$ compared to other trials. 1BL/1RS class increased thousand-grain weight, SPAD unit and awn length. The negative effect of $1 \mathrm{BL} / 1 \mathrm{RS}$ translocation on grain yield is an undesirable characteristic in hot and dry environments. Thus, based on our results, the $1 \mathrm{BL} / 1 \mathrm{RS}$ translocation may not convey an advantage for SeriM82/Babax population in heat and drought stress conditions. It is suggested to limit the use of $1 \mathrm{BL} / 1 \mathrm{RS}$ translocation lines only as a source of genetic variation. 


\section{Acknowledgement}

The authors gratefully acknowledge Dr. Lynne McIntyre from CSIRO plant industry for providing the molecular map and rye-specific markers scores of SeriM82/Babax population.

\section{References}

Bagherikia, S., Karimzadeh, G., Naghavi. M.R. 2014. Distribution of 1AL.1RS and 1BL.1RS wheat-rye translocations in Triticum aestivum using specific PCR. Biochem. Syst. Ecol. 55:20-26.

Bartos, P., Valkoun, J., Kosner, J., Skovencikova, U. 1973. Rust resistance of some European wheat cultivars derived from rye. Proc. $4^{\text {th }}$ Inter. Wheat Genet. Symp. Univ. of Missouri Press. Columbia, MO, USA. pp. $145-146$.

Borrás, L., Slafer, G.A., Otegui, M.E. 2004. Seed dry weight response to source-sink manipulations in wheat, maize, soybean. A quantitative reappraisal. Field Crops Res. 86:131-146.

Calderini, D.F., Savin, R., Abeledo, L.G., Reynolds, M.P., Slafer, G.A. 2001. The importance of the immediately preceding anthesis period for grain weight determination in wheat. Euphytica 119:199-204.

Carver, B.F., Rayburn, A.L. 1994. Comparison of related wheat stocks possessing 1B or 1RS.1BL chromosomes: Agronomic performance. Crop Sci. 34:1505-1510.

Churchill, G.A., Doerge, R.W. 1994. Empirical threshold values for quantitative trait mapping. Genetics 138:963-971.

Clarke, J.M., McCaig, T.N., DePauw, R.M. 1993. Relationship of glaucousness and epicuticular wax quantity of wheat. Canad. J. Plant Sci. 73:961-967.

Dadkhodaie, N.A., Singh, D., Park, R.F. 2011. Characterization of resistance to leaf rust in an international bread wheat nursery. J. Plant Pathol. 93:627-641.

Dhaliwal, A.S., MacRitchie, F. 1990. Contributions of protein fractions to dough handling properties of wheatrye translocations cultivars. J. Cereal Sci. 12:113-122.

Doerge, R.W., Churchill, G.A. 1996. Permutation tests for multiple loci affecting a quantitative character. Genetics 142:285-294.

Ehdaie, B., Whitkus, R.W., Waines, J.G. 2003. Root biomass, water-use efficiency, and performance of wheatrye translocations of chromosomes 1 and 2 in spring bread wheat 'Pavon'. Crop Sci. 43:710-717.

Fenn, D., Lukow, O.M., Bushuk, W., DePauw, R.M. 1994. Milling and baking quality of 1BL/1RS translocation wheats. I. Effects of genotype and environment. Cereal Chem. 71:189-195.

Hoffmann, B. 2008. Alteration of drought tolerance of winter wheat caused by translocation of rye chromosome segment 1R. Cereal Res. Commun. 36:269-278.

Jiang, Q.T., Wei, Y.M., Andre, L., Lu, Z.X., Pu, Z.E., Peng, Y.Y., Zheng, Y.L. 2010. Characterization of omega secalin genes from rye, triticale and a wheat 1BL/1RS translocation line. J. Appl. Genet. 51:403-411.

Lopes, M.S., Reynolds, M.P., McIntyre, C.L., Mathews, K.L., Jalal Kamali, M.R., Mossad, M., Feltaous, Y., Tahir, I.S.A., Chatrath, R., Ogbonnaya, F., Baum, M. 2013. QTL for yield and associated traits in the SeriM82/Babax population grown across several environments in Mexico, in the West Asia, North Africa, and South Asia regions. Theor. Appl. Genet. 126:971-984.

Mathews, K.L., Malosetti, M., Chapman, S., McIntyre, L., Reynolds, M., Shorter, R., Van Eeuwijk, F. 2008. Multi-environment QTL mixed models for drought stress adaptation in wheat. Theor. Appl. Genet. 117:1077-1091.

McIntosh, R.A. 1983. A catalogue of gene symbols for wheat. In: Sakamoto, S. (ed.), Proc. $6^{\text {th }}$ Int. Wheat Genet. Symp. Kyoto Univ. Kyoto, Japan. pp. 1197-1255.

McIntosh, R.A., Bariana, H.S., Park, R.F., Wellings, C.R. 2001. Aspects of wheat rust research in Australia. Euphytica 119:115-120.

McIntyre, L.C., Mathews, K.L., Rattey, A., Chapman, S.C., Drenth, J., Ghaderi, M., Reynolds, M., Shorter, R. 2010. Molecular detection of genomic regions associated with grain yield evaluated under irrigated and rainfed conditions. Theor. Appl. Genet. 120:527-541. 
Monneveux, P., Reynolds, M.P., Zahravia, M., Mujeeb-Kazi, A. 2003. Effect of T1BL.1RS chromosome translocation on bread wheat grain yield and physiological related traits in a warm environment. Cereal Res. Commun. 31:371-378.

Moreno-Sevilla, B., Baenzinger, P.S., Peterson, C., Graybosch, R.A., McVey, D.V. 1995. The 1BL/1RS translocation: Agronomic performance of F3-derived lines from a winter wheat cross. Crop Sci. 35:1051-1055.

Olivares-Villegas, J.J., Reynolds, M.P., McDonald, G.K. 2007. Drought adaptive attributes in the SeriM82/ Babax hexaploid wheat population. Funct. Plant Biol. 34:189-203.

O’Toole, J.C., Moya, T.B. 1978. Genotypic variation in maintenance of leaf water potential in rice. Crop Sci. 18:873-876.

Pask, A.J.D., Pietragalla, J., Mullan, D.M., Reynolds, M.P. 2012. Physiological breeding II: A field guide to wheat phenotyping. CIMMYT, Mexico, D.F., Mexico. 140 p. ISBN 978-970-648-182-5.

Payne, R.W., Harding, S.A., Murray, D.A., Soutar, D.M., Bird, D.B. 2012. The 12 Guide to the GenStat Release 15, VSN International. Hemel Hempstead, UK.

Peake, A.S. 2003. Inheritance of Grain Yield and Effect of the 1BL/1RS Translocation in Three Bi-Parental Wheat (Triticum aestivum L.) Populations in Production Environments of North Eastern Australia. School of Land and Food Sciences, The University of Queensland. Brisbane, Australia. 204 p.

Peake, A.S., Gilmour, A., Cooper, M. 2011. The 1BL/1RS translocation decreases grain yield of spring wheat germplasm in low yield environments of north-eastern Australia. Crop Past. Sci. 62:276-288.

Pinto, R.S., Reynolds, M.P., Mathews, K.L., McIntyre, C.L., Olivares-Villegas, J.J., Chapman, S.C. 2010. Heat and drought adaptive QTL in a wheat population designed to minimize confounding agronomic effects. Theor. Appl. Genet. 121:1001-1021.

Rabinovich, S.V. 1998. Importance of wheat-rye translocations for breeding modern cultivars of Triticum aestivum L. Euphytica 100:323-340.

Rattey, A., Shorter, R., Chapman, S.C., Dreccer, M.F., Van Herwaarden, A.F. 2009. Variation for biomass and grain components, and traits conferring improved yield and grain weight, in an elite recombinant inbred wheat population grown under variable drought conditions. Aust. J. Agric. Res. 60:717-729.

Rajaram, S., Mann, C.E., Oniz-Ferrara, G., Mujeeb-Kazi, A. 1983. Adaptation, stability and high yield potential of certain 1B/1R CIMMYT wheats. In: Sakamoto, S. (ed.), Proc. of the $6^{\text {th }}$ Intern. Wheat Genet. Symp. Kyoto, Japan. pp. 613-621.

Rogowsky, P.M., Sorrela, M.E., Shepherd, K.W., Langridge, P. 1993. Characterization of wheat-rye recombinants with RFLP and PCR probes. Theor. Appl. Genet. 85:1023-1028.

Schlegel, R., Korzun, V. 1997. About the origin of 1RS.1BL wheat-rye chromosome translocations from Germany. Plant Breed. 116:537-540.

Singh, N.K., Shepherd, K.W., McIntosh, R.A. 1990. Linkage mapping of genes for resistance to leaf, stem and tripe rusts and $\omega$-secalins on the short arm of rye chromosome 1R. Theor. and Appl. Genet. 80:609-616.

Ugarte, C., Calderini, D.F., Slafer, G.A. 2007. Grain weigh, grain number responsiveness to pre-anthesis temperature in wheat, barley, triticale. Field Crops Res. 100:240-248.

Villareal, R.L., Rajaram, S., Mujeeb-Kazi, A., del Toro, E. 1991. The effect of chromosome 1B/1R translocation on the yield potential of certain spring wheats (Triticum aestivum L.). Plant Breed. 106:77-81.

Villareal, R.L., Mujeeb-Kazi, A., Rajaram, S., del Toro, E. 1994. Associated effects of chromosome 1B/1R translocation on agronomic traits in hexaploid wheat. Breed Sci. 44:7-11.

Villareal, R.L., del Toro, E., Mujeeb-Kazi, A., Rajaram, S. 1995. The 1BL/1RS chromosome translocation effect on yield characteristics in a Triticum aestivum L. cross. Plant Breed. 114:497-500.

Voorrips, R.E. 2002. MapChart: software for the graphical presentation of linkage maps and QTLs. Heredity 93:77-78.

Wang, S., Basten, C.J., Zeng, Z.B. 2007. Windows QTL Cartographer V2.5 user manual. Bioinformatic Research Center, North Carolina State University. Raleigh, NC, USA.

Zeller, F.J. 1973. 1B/1R wheat-rye chromosome substitutions and translocations. In: Sears, E.R., Sears, L.M.S. (eds), Proc. $4^{\text {th }}$ Intern. Wheat Genet. Symp., Univ, Missouri, Columbia, USA, pp. 209-221.

Zhao, C., Cui, F., Wang, X., Shan, S., Li, X., Bao, Y., Wang, H. 2012. Effects of 1BL/1RS translocation in wheat on agronomic performance and quality characteristics. Field Crops Res. 127:79-84. 
Zhou, Y., He, Z.H., Liu J.J., Liu, L. 2003. Distribution of 1BL/1RS translocation in Chinese winter wheat and its effect on noodle quality. In: Pogna, N.E., Romano, M., Pogna, E., Galterio, G. (eds), Proc. $10^{\text {th }}$ Intern. Wheat Genetics Symposium. Paestum, Italy. 3:1419-1421.

\section{Electronic Supplementary Material (ESM)}

Electronic Supplementary Material (ESM) associated with this article can be found at the website of CRC at http://www.akademiai.com/content/120427/

Electronic Supplementary Table S1. Maximum likelihood results of mixed model for agronomic, morphological and physiological characteristics in 1BL/1RS and 1B lines in eight environments

Electronic Supplementary Figure S1. The map of QTLs in translocation-harboring region of chromosome 1B 